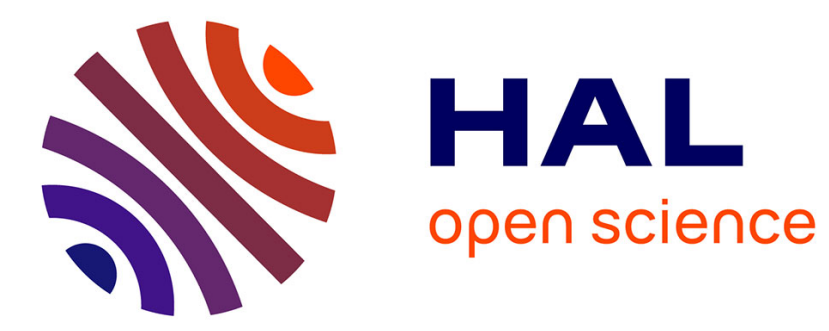

\title{
Power Dissipation in Reverberation Chamber Metallic Surfaces Based on Ferrous Materials
}

Andréa Cozza, Florian Monsef

\section{To cite this version:}

Andréa Cozza, Florian Monsef. Power Dissipation in Reverberation Chamber Metallic Surfaces Based on Ferrous Materials. IEEE Transactions on Electromagnetic Compatibility, 2018, 10.1109/TEMC.2018.2873657 . hal-01884750

\section{HAL Id: hal-01884750}

https://hal-centralesupelec.archives-ouvertes.fr/hal-01884750

Submitted on 1 Oct 2018

HAL is a multi-disciplinary open access archive for the deposit and dissemination of scientific research documents, whether they are published or not. The documents may come from teaching and research institutions in France or abroad, or from public or private research centers.
L'archive ouverte pluridisciplinaire $\mathbf{H A L}$, est destinée au dépôt et à la diffusion de documents scientifiques de niveau recherche, publiés ou non, émanant des établissements d'enseignement et de recherche français ou étrangers, des laboratoires publics ou privés. 


\title{
Power Dissipation in Reverberation Chamber Metallic Surfaces Based on Ferrous Materials
}

\author{
Andrea Cozza, Senior Member, IEEE, Florian Monsef, Member, IEEE
}

\begin{abstract}
Models predicting the composite quality factor (QF) of a reverberation chamber (RC) consider, among several potential contributors, dissipation in its metallic boundaries. The related partial quality factor, $Q_{w}$, is of fundamental importance, as it controls the asymptotic high-frequency behavior of an $\mathrm{RC}$ and, ultimately, its ability to generate high-intensity fields. Yet, the current model has been known to overestimate in certain cases the composite QF by up to several $\mathrm{dB}$. This paper provides insight into the causes of these disagreements by introducing generalized models of dissipation in ferrous materials found in RCs, by first acknowledging that their magnetic permeabilities are complex quantities, which is shown to theoretically boost dissipation well beyond the $\mathrm{GHz}$ range. A more general dissipation model is presented, taking into account the layered nature of steel plates. Among its predictions, confirmed by experiments, are extra losses from steel surfaces in the lower frequency range and the linear increase in $Q_{w}$ over the $\mathbf{G H z}$ range, as opposed to a squareroot dependence expected for homogeneous bulk metals. Metallic coating layers, originally introduced to protect steel plates, have therefore a more fundamental role to play, controlling dissipation levels by reducing interactions with ferrous materials and should therefore be designed accordingly.
\end{abstract}

Index Terms-Reverberation chamber, quality factor, metallic boundaries, magnetic permeability.

\section{INTRODUCTION}

$\mathbf{T}$ HEORETICAL models of average composite quality factor (QF) in reverberation chambers (RCs) explain overall energy loss by breaking it down into individual contributions [1]. For an unloaded RC, these basically amount to power losses through antennas and Joule dissipation in metallic structures, mostly coming as plates making up an RC boundaries, e.g., walls. Theory predicts that wall losses dominate the QF in the higher frequency range, imposing an asymptotic behavior expected to scale as the square root of the frequency. The main features of the QF predicted by theory are indeed broadly supported by experimental results, but non-negligible disagreements have been reported in the past, e.g., [1], [2], for ferrous structures. Steel is indeed a typical choice for building $\mathrm{RCs}$, since it has good mechanical properties and relatively low costs, compared to conductors such as aluminum and copper, which are better conductors and have a negligible magnetic behavior, but are handicapped by a relatively low Young modulus (i.e., stiffness) and higher costs.

Observations available in the literature basically point to two issues: an overestimation of the QF when using typical conductivity values for wall metals and disagreements in the frequency dependence of the QF [3]. While a number

A. Cozza and F. Monsef are with PIEM, GeePs (UMR 8507), 11 rue Joliot-Curie, 91192 Gif-sur-Yvette, France. Contact e-mail: andrea.cozza@ieee.org. of reasons could explain disagreements between theory and experimental results, it is important to notice that the above mentioned theoretical models require an accurate knowledge of parameters such as electrical conductivity and magnetic permeability of the metals involved. In practice, these quantities are not easily accessible, and the values actually used for theoretical models are at best educated guesses, not necessarily supported by experimental evidence or results available in the literature.

This paper endeavours to show that these discrepancies have two causes. First, we discuss what appears to be a misconception about the high-frequency magnetic behavior of ferrous materials, which has sometimes been assumed within the EMC community to be negligible at microwave frequencies. Second, we argue about the fundamental role played by metal coatings, mostly used for galvanic protection of steel and iron plates. The resulting layered structure is here shown to have an effective response very different from either metal when considered in bulk form.

The paper starts with a concise survey of the properties of ferrous conductors, presented in Sec. II, in order to avoid unrealistic assumptions and serve as a basis for the definition of a realistic model and the interpretation of experimental results. In particular, the fundamental contribution of resistive magnetic permeability at microwave frequencies, introduced in Sec. III, appears to be unacknowledged in the context of reverberation chambers. As a consequence, Sec. IV extends the current dissipation model for metallic surfaces in order to describe more realistically dissipation mechanisms. Given the practical impossibility of predicting the physical parameters of steel plates, Sec. V introduces an estimator of the apparent conductivity of metallic surfaces in an RC, based on its composite QF. Sec. VI shows how this information, jointly with the proposed layered model, yields effective thicknesses of the metallic coating that are consistent with common manufacturing choices. Predicted effects, such as the linear evolution of the QF and higher-than-expected losses in the lower frequency range are supported by experiments and results available in the literature, reported in Sec. VII. Finally, Sec. VIII discusses how the proposed model can be applied in designing optimal metallic coatings that would reduce interactions with background ferromagnetic materials (e.g., steel plates), estimating the minimum thickness for non-magnetic metallic coatings, in order to still benefit from the mechanical properties of steel plates while avoiding their excessive losses, rather than entirely switching to more expensive, and mechanically less effective, metals such as aluminum.

The main conclusion from this study is that steel coatings need to be chosen carefully, not just as anti-corrosion protec- 
tions, but mainly as they act by attenuating the ferromagnetic behavior (and losses) of steel plates, while also providing potentially better conductive surfaces where dissipation can be lowered with respect to the case of steel, which should only have a mechanical/ structural role in an RC.

In the rest of this paper dependence from the frequency variable $\nu$ is mostly explicit, but may be omitted for the sake of brevity. More importantly, all derivations are based on the usual assumption of diffusive energy propagation, which therefore requires due care in the interpretation of experimental results.

\section{AN OVERVIEW OF STEEL PLATES}

This section presents a short survey of information that has a direct impact on the way steel plates reflect and dissipate electromagnetic energy. Results from the literature explaining strong dissipation at microwave frequencies in ferrous metals are summarized, seemingly not well-known to the EMC community, contradicting simplistic assumptions about these complex materials. The current unavailability of theoretical models explaining dispersion in ferromagnetic behavior is discussed. Together with the high variability in the characteristics of iron alloys, these results suggest an impossibility of accurately predicting power losses in steel plates. Furthermore, the solutions currently used to passivate the surface of ferrous metals are briefly summarized, adding further uncertainty to their electromagnetic response.

\section{A. Steel composition}

The word steel can mistakenly be taken as referring to a family of materials with similar composition and properties. The opposite rather holds, as there exist hundreds of typologies of steel, mainly depending on the mechanical properties required for a given application [4, InfoNote 1.8]. While steel is predominantly composed of iron, the latter is too ductile to be used in most applications. All ferrous materials are alloys of iron and other elements, the most important is arguably carbon, forming the basis for all steels. An important point for later discussions is to acknowledge the fact that very small variations in the fraction of carbon can lead to significant modifications in the mechanical properties of a steel class, making it pass from a formable material for carbon fractions below $0.3 \%$, to a hardly machinable material for a little more than $0.6 \%$. This should not be dismissed as having only an impact on steel's mechanical properties, since the presence of carbon atoms directly impacts the crystal lattice of steel, thus effectively impacting all properties related to it, including electrical conductivity and magnetic permeability, as summarized in [5]. Other alloy elements are also included, like chromium, silicon, etc. leading to a large spectrum of possible combinations. As a result, materials labelled as steel may have any of a number of properties, hardly predictable, unless its specific typology is known from the start [6].

\section{B. Magnetic permeability}

Steel's ability to respond with strong magnetization levels when submitted to magnetic fields is know as ferromagnetism, displaying relative magnetic permeabilities $\mu_{r}$ well above unity. Data are widely available for DC and very low frequencies, typically well below the $\mathrm{kHz}$ range. Ferromagnetic properties are of interest in EMC for shielding at low frequencies [7], [8]. Most reference books dealing with EMC shielding take the position of focusing on using steel for shielding at low frequencies, where its high permeability allows a steep reduction of the skin depth and therefore to reduce a shielding thickness. This is no longer an issue as frequencies reach the RF region, with skin depths well below a millimeter. Surprisingly, most EMC books neither cite references dealing with direct measurements [7]-[13] nor provide the frequency at which the suggested value applies; [14] alone insists on the extreme variability of steel properties. Moreover, it is also often postulated that a swift reduction in ferromagnetic responses takes place at higher frequencies, often stating that $\mu_{r} \simeq 1$ in proximity of the $\mathrm{GHz}$ region. As discussed below, this assumption is only correct for the real part of $\mu_{r}$, but neglects the fundamental role of its imaginary part.

As most RCs are made of steel, the problem of knowing what value of permeability should be used appears when dealing with predicting their QF [15, Sec. 3.1.1]. The common assumption of $\mu_{r} \simeq 1$ is also uniformly applied, to the best of our knowledge. A few exceptions exist [7, p. 264], [14, p. 514], where much higher values, e.g. $\mu_{r} \simeq 50$ at $1 \mathrm{GHz}$, are reported. It seems reasonable to conjecture that the inaccurate reporting of the high-frequency magnetic behavior of steels came about by the reduced need for high permeabilities in shieldings above the $\mathrm{MHz}$ range [6].

In fact, literature reports experimental data proving that ferromagnetism is not only still observable in the microwave range, but is actually remarkably high at much higher frequencies. The study of the magnetic response of steel was a major issue in the 30's and 40's in the twentieth century, as the nascent waveguide research community focused on predicting dissipation losses in ferrous waveguides. Of interest for QF in RCs, [16] presented an example of underestimated attenuation due to neglecting permeability and surface roughness. A resistive (defined below) relative permeability of 250 is also there reported for mild steel at $10 \mathrm{GHz}$, as derived in previous work and is argued to be likely underestimated. The approach is similar to the one adopted in the present paper, i.e., to estimate permeability from power attenuation, which was then argued to have good accuracy. [17] extended these results to $27 \mathrm{GHz}$ and showed amplitudes of resistive $\mu_{r}$ up to 84 for mild steel.

A fundamental issue throughout this paper is the complex nature of $\mu_{r}=\mu_{r}^{\prime}-\mathrm{j} \mu_{r}^{\prime \prime}$, a counterintuitive idea first suggested by W. Arkadiew in 1926. Experimental evidence of the soundness of this description was presented in a number of papers where ferromagnetic effects were observed both on the resistance of inductors containing samples of ferrous materials as their core, as well as in their self-inductance; see [19] for a concise explanation and an overview of previously published results. Two values of relative permeability were therefore estimated, referred to as $\mu_{R}$ and $\mu_{L}$. The existence of two different coefficients was later proven to be consistent with the idea of a complex permeability, with $\mu_{R}$ quantifying magnetic 

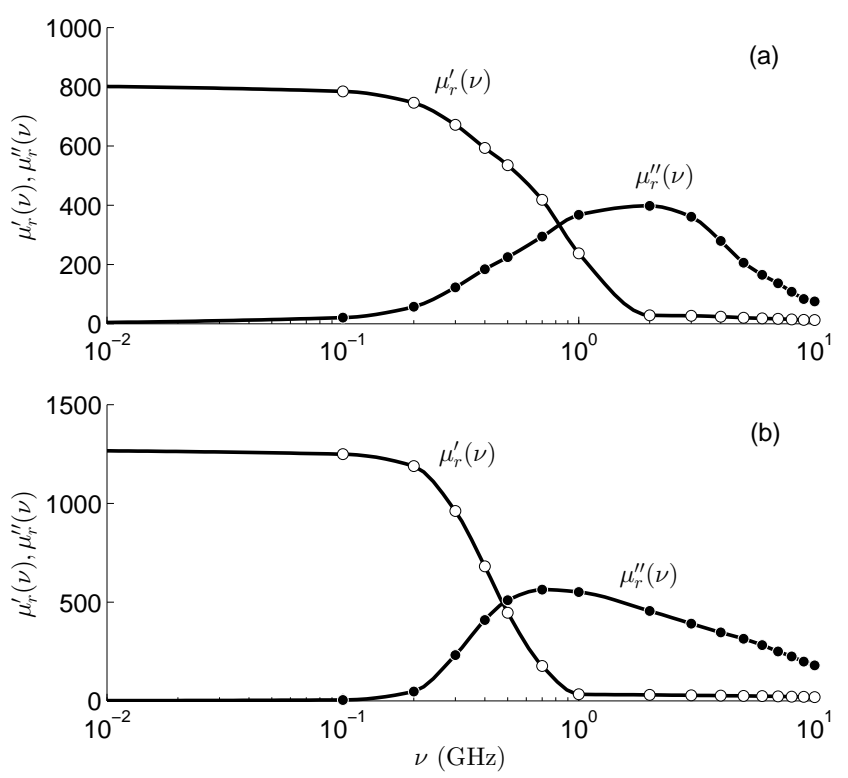

Fig. 1: Complex relative magnetic permeability $\mu_{r}(\nu)$ for : (a) bright iron and (b) medium-carbon steel, or mild steel, elaborated from data reported by R. Millership et al. in [18] and interpolated with cubic functions.

losses, hence its being referred to as resistive permeability. One of the most detailed experimental studies of high-frequency permeability in irons and steels can be found in [18], together with conversion formulae between $\left\{\mu_{R}, \mu_{L}\right\}$ and $\left\{\mu_{r}^{\prime}, \mu_{r}^{\prime \prime}\right\}$. Results in Fig. 1 are obtained from data there reported and show that indeed $\mu_{r}^{\prime}$ has a sharp transition to lower values around $1 \mathrm{GHz}$, but it is still well above unity. At the same time, $\mu_{r}^{\prime \prime}$ takes a dominant role, with very high values reported well above $10 \mathrm{GHz}$; [19] states that the resistive permeability reduces by half around $3 \mathrm{GHz}$. The same Authors also cite infra-red experiments that showed that the ferromagnetic behavior subdued only above $10 \mathrm{THz}$, with $\mu_{r}^{\prime} \rightarrow 1$ and $\mu_{r}^{\prime \prime} \rightarrow 0$. A similar conclusion was reached in [20], together with the fact that magnetic hysteresis, a pervasive phenomenon in ferromagnetic materials, vanishes above $10 \mathrm{MHz}$. Also of interest are the theoretical relationships summarized in [21], showing how high DC values of permeability necessarily imply highfrequency magnetic losses. It seems therefore necessary to include the case of a complex $\mu_{r}$ in QF models for an RC, as discussed in Sec. III.

With the discussion at the beginning of this section in mind, it is ironic that the above results were surprising at the time as it was not clear what phenomena should make the magnetic permeability decrease over frequency. One of the first attempts at finding an explanation was proposed in [19], based on the idea that as skin penetration decreased, the number of magnetic domains taking part in the magnetization of a material would reduce till the point of concerning only fractions of the superficial domains. The resulting theory predicted a reduction of $\mu_{r}$, but also predicted magnetic resonances that were reported as not observed experimentally in [18]. Other attempts were proposed, by invoking differences in the superficial regions of a ferromagnetic material [20], together with relaxation models analogous to the Debye model for dielectric materials, as done by Becker. This latter model was shown in [18], [22] not to reproduce the actual frequency evolution of $\mu_{r}$, which was found to be slower than predicted. More accurate models were presented later, e.g., accounting for inter-domain friction, but were not considered completely satisfying. [23] concluded about the lack of accurate quantitative models for magnetic losses, in spite of the clear understanding of the physical mechanisms involved.

This brief overview is intended to make it clear that no simple model can predict the frequency evolution of permeability in steels, since it is not only affected by their chemical composition, but also by the kind of metallurgical and thermal processes they undergo, such as annealing and tempering; how the raw material is drawn, hot or cold, and so on, has an impact, too. All these processes have a direct impact on the distribution of magnetic domains. The only constant is the fact that $\left|\mu_{r}\right| \gg 1$ well above $10 \mathrm{GHz}$, a property exploited in Sec. IV in order to derive a simple model not requiring a precise knowledge of steel's permeability.

\section{Electrical conductivity}

The typical values found in the literature for the electrical conductivity of steels range from $1.6 \mathrm{MS} / \mathrm{m}$ for alloy steels, in particular stainless steel, up to $6 \mathrm{MS} / \mathrm{m}$ for cast steel. Values exceeding $10 \mathrm{MS} / \mathrm{m}$ have been reported for purer forms of iron. These values refer to bulk forms and are typically obtained at DC. Results at higher frequency, in particular in the microwave range are available but require a careful interpretation, since the contributions of finite electrical conductivity and ferromagnetic behavior cannot be measured separately [17], [24], thus requiring assumptions. Results from non-magnetic metals suggest that the bulk conductivity is practically constant [24], with transitions rather expected at near-optical frequencies.

Apparent frequency-dependence in the conductivity of specimens with finite thickness was explained by the roughness of their surfaces. Relevant studies are [16]-[19], [24], [25], where the precision of machined surfaces was shown to impact the high-frequency conductivity and therefore power loss in waveguides. References cited in those paper provide the theoretical basis for understanding how roughness impacts highfrequency conductivity. Those results prove that the effective conductivity may be reduced at most by a factor 2 , with values around 1.6 more likely according to [25]. These reduction factors will be shown in Sec. VI to be insufficient to explain much stronger reductions in the apparent conductivity in steel plates, thus pointing to ferromagnetic losses as the most likely reason for increased dissipation.

\section{Surface finishes and coatings}

Unless stainless steel is used, which is unlikely due to its higher cost, poorer conductivity, welding and structural strength, standard steels need a coating layer in order to protect them against corrosion. Protective coatings include paints, plating with pure metals (e.g., copper, zinc, etc.) or galvanic 
protection with zinc and its alloys. This last option is by far the less expensive both at manufacturing and maintenance levels.

Zinc enables protection thanks to a passivation layer of zinc oxide of sub-micron thickness, and galvanic protection ensured by the underlying zinc-rich layer, as an element more reactive than iron. The thickness of the coating layer changes according to the severity of the environment where steel parts will be mounted, and on its required service life without noticeable corrosion [6], [26], [27]. For indoor applications (category C1 in [26]) an average corrosion $<0.1 \mu \mathrm{m} /$ year is expected, implying that a $3 \mu \mathrm{m}$ zinc coating would last for at least 30 years. Pure zinc $(98 \%)$ is the most common option for unpainted plates in indoor conditions, while iron-zinc alloys eventually annealed are considered when paint is applied. Other alloys with aluminum (e.g., $\mathrm{GalFan}^{(}{ }^{\mathrm{C}}$, ZincAl ${ }^{\circledR}$ ) are also available but more costly as they ensure higher-level corrosion protection unnecessary for indoor applications.

Zinc coatings are usually, but not only, applied to steel sheets either by means of electro-plating or continuous-sheet galvanization [27]. Other mechanical processes also exist, but are unlikely for steel plates. These two coating technologies produce rather different morphologies and compositions, as apparent from the micrographies in [27]. Coatings obtained with electro-plating are typically below $7 \mu \mathrm{m}$, while continuous-sheet galvanization yields layers easily thicker than $100 \mu \mathrm{m}$ [28]. The latter option demands a closer analysis, as the coating is actually composed of 4 sub-layers, made of iron-zinc alloys in varying proportions caused by iron diffusion, of which only the superficial one can be regarded as containing almost pure zinc [29], [30]. In both cases, for indoor applications, the actual zinc layer, i.e., the most external one, is typically below $5 \mu \mathrm{m}$ thick, with the G01 class for indoor environments displaying thicknesses within 0.42-2.1 $\mu \mathrm{m}$.

Visual aspects of coatings obtained with the two procedures can be very similar, but the presence of spangled spots is a clear indication of hot-dip galvanization. There is no obvious way of telling what coating was applied to a steel plate, adding to the uncertainty of the properties of a steel plate.

Sec. IV shows how a metal coating acts by attenuating the magnetic response of underlying steel surfaces. It is therefore of importance to know the properties of zinc coatings. Electrical conductivity will depend on the fraction of zinc involved. According to [31], the residual resistivity of zinc contaminated by a $10 \mathrm{ppm}$ concentration of $\mathrm{Fe}$ atoms is about 100 times higher than that of pure zinc. Results in [32] show that the room-temperature resistivity of iron-zinc alloys increases approximatively linearly with the temperature. Hence, in the absence of more specific results, it seems reasonable to expect $\mathrm{ZnFe}$ alloys to present a room-temperature resistivity higher than that of pure bulk zinc ( $\sim 16 \mathrm{MS} / \mathrm{m}$ ), depending on $\mathrm{Fe}$ concentration.

Pure zinc is not magnetic, but iron-zinc alloys found in continuous-sheet galvanization are magnetic, as shown in [33], with a permeability linearly proportional to the iron fraction. As a result, the layer just after that made of almost pure zinc, where typically a $6 \%$ concentration of iron is found, a substantially higher magnetic permeability can be expected, with $\left|\mu_{r}\right| \gtrsim 20$. These facts will be important in the interpretation of results shown in Sec. VI. The common thread between the two galvanization technologies is the presence of a superficial layer of almost pure zinc across a few microns, on top of a magnetic substrate, either bulk steel or zinc-iron alloys.

\section{DISSIPATION IN BULK FERROUS BOUNDARIES}

In light of the information summarized in Sec. II-B, a nonnegligible ferromagnetic behavior must be expected for steelbased RC surfaces. Models predicting individual contributions to power loss in an RC were discussed in [1], among which dissipation in metallic surfaces (e.g., walls) and through antennas. These contributions are usually modeled under the shape of partial quality factors $\left\{Q_{i}(\nu)\right\}$, contributing to the overall composite quality factor $Q(\nu)$, as

$$
Q^{-1}(\nu)=\sum_{i=1}^{N_{Q}} Q_{i}^{-1}(\nu),
$$

with $N_{Q}$ the number of such contributions. In the following, $Q_{w}$ will refer to the partial quality factor explaining boundaryrelated losses in an RC with an internal volume $V$, with metallic boundaries covering a surface $S$.

The original derivation of $Q_{w}$ presented in [1] considered the general case of magnetic surfaces, but the final expression there derived cannot be applied to the case of a complex magnetic permeability, since it would result into a complex quality factor. For this reason, this section extends the derivation in [1] to a more general configuration.

Dissipation in metallic surfaces was modelled in [1] by first computing the reflection coefficients for TE $(\perp)$ and TM $(\|)$ incidences [34]

$$
\begin{aligned}
\Gamma^{\perp}(\nu) & =\frac{\mu_{2} k_{1} \cos \vartheta_{i}-\mu_{1} \sqrt{k_{2}^{2}-k_{1}^{2} \sin ^{2} \vartheta_{i}}}{\mu_{2} k_{1} \cos \vartheta_{i}+\mu_{1} \sqrt{k_{2}^{2}-k_{1}^{2} \sin ^{2} \vartheta_{i}}} \\
\Gamma^{\|}(\nu) & =\frac{\mu_{1} k_{2}^{2} \cos \vartheta_{i}-\mu_{2} k_{1} \sqrt{k_{2}^{2}-k_{1}^{2} \sin ^{2} \vartheta_{i}}}{\mu_{1} k_{2}^{2} \cos \vartheta_{i}+\mu_{2} k_{1} \sqrt{k_{2}^{2}-k_{1}^{2} \sin ^{2} \vartheta_{i}}}
\end{aligned}
$$

where a plane wave impinges on medium 2 (metal) from medium 1 (air), with an angle of incidence $\vartheta_{i} ; k_{p}$ and $\mu_{p}$, for $p=1,2$, are the wavenumbers and relative magnetic permeabilities of the two media. In the context of this section, metal-related quantities will be represented by a $m$ subscript, while those for air will use an $o$.

These formulae can be approximated for the case of well conducting metallic surfaces, for which $\left|k_{2}\right| \gg\left|k_{1}\right|$, as

$$
\begin{aligned}
\Gamma^{\perp}(\nu) & \simeq-1+2 \cos \vartheta_{i} \sqrt{\mathrm{j} \omega \epsilon_{o} q_{m}(\nu)} \\
\Gamma^{\|}(\nu) & \simeq 1-2\left(\cos \vartheta_{i}\right)^{-1} \sqrt{\mathrm{j} \omega \epsilon_{o} q_{m}(\nu)}
\end{aligned}
$$

with

$$
q_{m}(\nu)=\mu_{m}(\nu) / \sigma_{m}(\nu),
$$

the only metal-related quantity in (3). As discussed, the general case $\mu_{m}(\nu) \in \mathbb{C}$ needs to be addressed, with $\mu_{m}(\nu)=$ $\mu_{m}^{\prime}(\nu)-\mathrm{j} \mu_{m}^{\prime \prime}(\nu)$, where $\mu_{m}^{\prime}, \mu_{m}^{\prime \prime}>0$ for steels, but not 


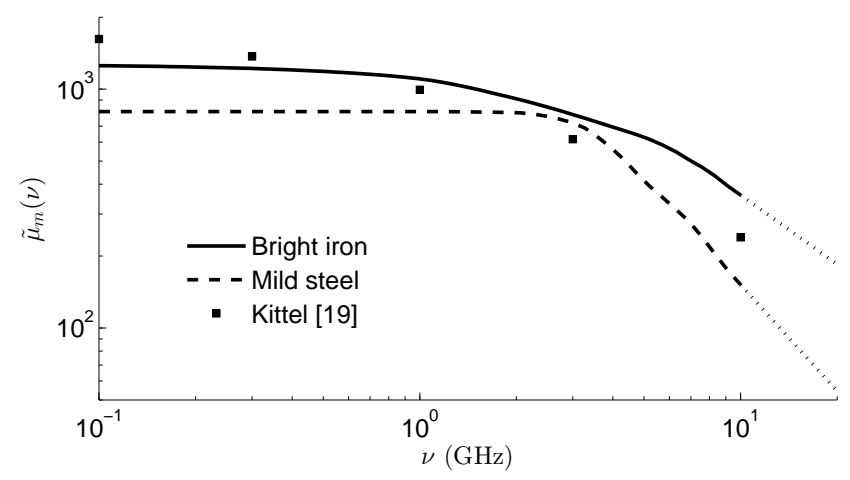

Fig. 2: Effective relative magnetic permeability $\tilde{\mu}_{m}(\nu)$, as defined by (9), for Millership's data [18] and for Kittel's regression curves [19]. Dotted portions represent mathematical continuation of experimental data.

necessarily for other composite materials characterized by magnetic resonances, such as ferrites [35]. The complex nature of $q_{m}(\nu)$ is a consequence of including $\mu_{m}^{\prime \prime}(\nu)$, a fundamental operation since it dominates the high frequency response of soft ferromagnetic materials, as recalled in Sec. II-B.

Hill's derivation in [1] calls for the computation of the square modulus of the reflection coefficients (3). Two relationships are here useful: a) for a complex number $x,|1+x|^{2} \simeq$ $1+2 \operatorname{Re} x$, for $|x| \ll 1$, and b) $\operatorname{Re} \sqrt{x}=\sqrt{(|x|+\operatorname{Re} x) / 2}$. Following step-by-step Hill's derivation, the more general case of lossy magnetic materials straightforwardly results into

$$
Q_{w}(\nu)=\frac{3}{2} \frac{V}{S} \sqrt{\frac{\pi \nu \mu_{o}}{\tilde{q}_{m}(\nu)}}=\frac{3}{2} \frac{V}{t_{m}(\nu) S},
$$

where

$$
\tilde{q}_{m}(\nu)=\left|q_{m}(\nu)\right|-\operatorname{Im} q_{m}(\nu)
$$

and

$$
t_{m}(\nu)=\sqrt{\tilde{q}_{m}(\nu) / \pi \nu \mu_{o}},
$$

is the effective dissipation thickness ${ }^{1}$.

Eqs. (5) and (6) can also be alternatively interpreted by introducing the effective conductivity

$$
\tilde{\sigma}_{m}(\nu)=1 / \tilde{q}_{m}(\nu) .
$$

Since the conductivity $\sigma_{m}(\nu)$ of good conductors can be approximated by a real number, (6) leads to the definition of the effective permeability $\tilde{\mu}_{m}(\nu)$

$$
\tilde{\mu}_{m}(\nu)=\tilde{q}_{m}(\nu) \sigma_{m}(\nu)=\left|\mu_{m}(\nu)\right|-\operatorname{Im} \mu_{m}(\nu),
$$

which coincides with the resistive relative permeability [17].

Applying similar steps, the skin depth $\delta_{m}$ of a magnetic conductor should be updated as

$$
\delta_{m}(\nu)=1 / \sqrt{\pi \nu \mu_{o} \tilde{\mu}_{m}(\nu) \sigma_{m}(\nu)},
$$

hence

$$
t_{m}(\nu)=\delta_{m}(\nu) \tilde{\mu}_{m}(\nu),
$$

${ }^{1}$ Eq. (6) can also be stated as $\left|q_{m}(\nu)\right|(1-\sin \alpha(\nu))$, with $\alpha(\nu)$ the angle of the complex quantity $q_{m}(\nu)$

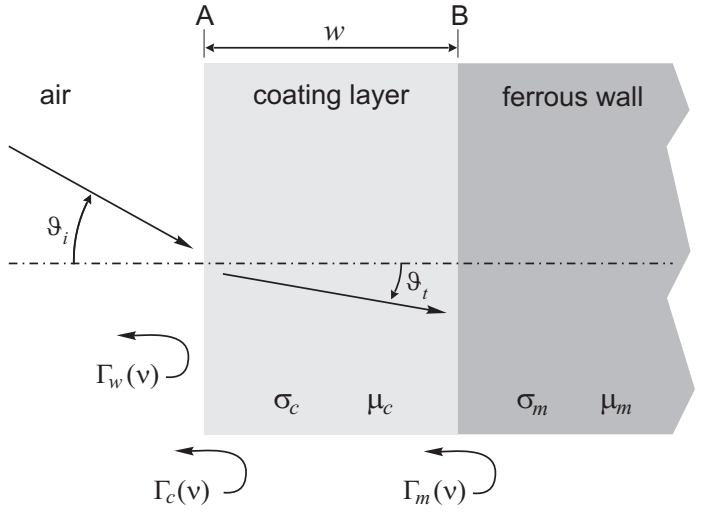

Fig. 3: Cross-section model used in the derivation of the effective reflectivity $\Gamma_{w}$ of metal-coated surfaces. The reflection coefficients $\Gamma_{c}$ and $\Gamma_{m}$ are to be interpreted as local or surge reflections, i.e., for half-space configurations across interfaces $\mathrm{A}$ and $\mathrm{B}$.

which proves that $t_{m}(\nu)>\delta_{m}(\nu)$, potentially by several orders of magnitude, and thus that the skin depth should not be regarded as an estimate of the effective thickness of metal where dissipation occurs.

Fig. 2 presents the results obtaining by applying (9) to Millership's data (cf. Fig. 1), where the effective permeability $\tilde{\mu}_{m}(\nu)>100$ still at $10 \mathrm{GHz}$. Further results from Kittel [19] are also reported, approximating data collected from older literature. The three sets of data present very similar features, and would lead to considering that steel-based RCs should present an almost fifty-fold increase in their boundary-related dissipation with respect to assuming non-magnetic properties. No such large disagreement has ever been reported to our knowledge, with typical disagreements between theoretical and measured QFs rather smaller than a factor ten. The reasons for this unmet prediction is explained by the fundamental role played by metal coatings used in steel plates.

\section{Metal-COATED Plates: APPARENT CONDUCTIVITy}

In view of the common use of galvanized steel-plate surfaces (cf. Sec. II-D), it is necessary to extend the standard loss model (5) to include layered structures (cf. Fig. 3), including a metallic layer of thickness $w$, conductivity $\sigma_{c}(\nu)$ and relative magnetic permeability $\mu_{c}(\nu)$, potentially complex. While the coating thickness is finite, the background ferrous metal is assumed to be much thicker than its skin depth over the entire frequency range, thus effectively treating it as a bulk material.

Apparent reflectivities for TE and TM impinging waves now need to be derived for this layered structure. The local (or surge) reflectivities at the two interfaces A and B in Fig. 3, i.e., involving half-space bulk configurations, are referred to as $\Gamma_{c}(\nu)$ and $\Gamma_{m}(\nu)$ for the coating and the background metal interfaces, respectively. These reflectivities involve only the two materials across each interface and will serve as partial results in the computation of the equivalent reflectivities of the layered structure. 
According to (2) local reflectivities at the air-coating interface read

$$
\begin{aligned}
\Gamma_{c}^{\perp}(\nu) & =\frac{\sqrt{\mathrm{j} \omega \epsilon_{o} q_{c}(\nu)} \cos \vartheta_{i}-1}{\sqrt{\mathrm{j} \omega \epsilon_{o} q_{c}(\nu)} \cos \vartheta_{i}+1} \\
\Gamma_{c}^{\|}(\nu) & =\frac{1-\sqrt{\mathrm{j} \omega \epsilon_{o} q_{c}(\nu)} / \cos \vartheta_{i}}{1+\sqrt{\mathrm{j} \omega \epsilon_{o} q_{c}(\nu)} / \cos \vartheta_{i}}
\end{aligned}
$$

where $q_{c}(\nu)=\mu_{c}(\nu) / \sigma_{c}(\nu)$, with the subscript $c$ indicating coating parameters. Transmission through an interface between two half spaces must satisfy Snell's law

$$
k_{1} \sin \vartheta_{i}=k_{2} \sin \vartheta_{t},
$$

where $k_{p}, p=1,2$, refers to the wavenumbers of the two materials, with $k_{2}=k_{c} ; \vartheta_{t}$ is the angle of propagation of the wave transmitted through the coating. At the air-coating interface $\left|k_{c}\right| \gg\left|k_{1}\right|$, by several orders of magnitude at microwave frequencies, thus allowing to approximate $\sin \vartheta_{t} \simeq 0$, i.e., a practically normal incidence at the coating-metal interface. As a result, the local reflectivity there simplifies into

$$
\Gamma_{m}^{\perp}(\nu)=\frac{1-\sqrt{q_{c}(\nu) / q_{m}(\nu)}}{1+\sqrt{q_{c}(\nu) / q_{m}(\nu)}}
$$

where $q_{m}(\nu)$ is defined in (4), while $\Gamma_{m}^{\|}(\nu)=-\Gamma_{m}^{\perp}(\nu)$.

Apparent (or effective) reflection coefficients can now be computed by solving, for either TE or TM incidence, [36, Sec. 4.4.4]

$$
\Gamma_{w}(\nu)=\frac{\Gamma_{c}(\nu)+P^{2}(\nu) \Gamma_{m}(\nu)}{1+P^{2}(\nu) \Gamma_{c}(\nu) \Gamma_{m}(\nu)}
$$

where $P(\nu)=\exp \left[-\mathrm{j} k_{c}(\nu) w\right]$ models the propagation of a plane wave through the coating layer; (15) takes into account the series of inner reflections within the coating layer and partial transmissions back to air.

For the special case of galvanization, the above result can be simplified, since $\sigma_{c} / \omega \epsilon_{o} \gg 1$ well beyond the microwave region. As a result

$$
\Gamma_{w}^{\perp}(\nu) \simeq-1+2 \cos \vartheta_{i} \sqrt{\mathrm{j} \omega \epsilon_{o} q_{c}(\nu)} \frac{1+P^{2}(\nu) \Gamma_{m}(\nu)}{1-P^{2}(\nu) \Gamma_{m}(\nu)},
$$

which can in turn be expressed as

$$
\Gamma_{w}^{\perp} \simeq-1+2 \cos \vartheta_{i} \sqrt{\mathrm{j} \omega \epsilon_{o} q_{c}} \frac{1+\sqrt{q_{c} / q_{m}} \tanh \left(\mathrm{j} k_{c} w\right)}{\sqrt{q_{c} / q_{m}}+\tanh \left(\mathrm{j} k_{c} w\right)},
$$

where $k_{c}(\nu)=(1-\mathrm{j}) / \delta_{c}(\nu)$, with $\delta_{c}(\nu)=1 / \sqrt{\pi \nu \mu_{o} \mu_{c} \sigma_{c}}$ the skin depth for the coating layer.

Following the same procedure

$$
\Gamma_{w}^{\|}(\nu) \simeq 1-2 \frac{\sqrt{\mathrm{j} \omega \epsilon_{o} q_{c}}}{\cos \vartheta_{i}} \frac{1+\sqrt{q_{c} / q_{m}} \tanh \left(\mathrm{j} k_{c} w\right)}{\sqrt{q_{c} / q_{m}}+\tanh \left(\mathrm{j} k_{c} w\right)} .
$$

Eqs. (17) and (18) can now be expressed in terms of (3), by introducing the apparent $q_{a}(\nu)$

$$
q_{a}(\nu)=q_{c}(\nu)\left[\frac{1+\sqrt{q_{c}(\nu) / q_{m}(\nu)} \tanh \left(\mathrm{j} k_{c} w\right)}{\sqrt{q_{c}(\nu) / q_{m}(\nu)}+\tanh \left(\mathrm{j} k_{c} w\right)}\right]^{2} .
$$

As a consequence, (5) and (6) can still be employed, by simply updating $q_{m}(\nu) \rightsquigarrow q_{a}(\nu)$ together with definitions (8) and (9)
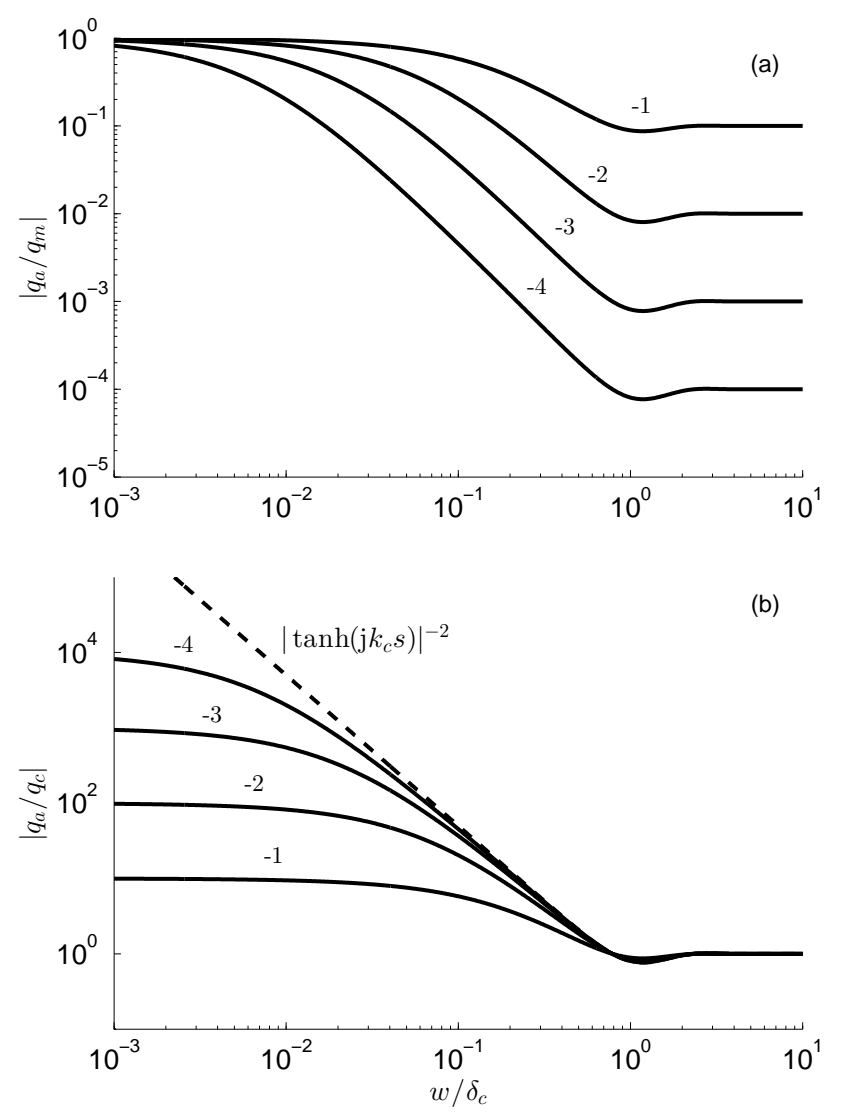

Fig. 4: Effective $q_{a}(\nu)$, as a function of the thickness $w$ of the coating layer and of the contrast in the metal conductivities, expressed as $\log \left|q_{c} / q_{m}\right|:$ (a) $\left|q_{a} / q_{m}\right|$ represents the apparent reduction in $q_{m}$ for the background metal, due to attenuation by the coating layer, while (b) $\left|q_{a} / q_{c}\right|$ is the apparent increase in $q_{c}$ for the coating layer, caused by interactions with the background ferromagnetic metal, giving raise to an apparent relative permeability $\mu_{a}$.

of the effective properties of the surface. Care must be taken in computing the square roots in the above expressions, in order to ensure that $\operatorname{Im} q_{a}(\nu)<0$, such that the operation (6) will lead to both real and imaginary parts of $q_{a}(\nu)$ contributing to dissipation losses.

Given that $\left|q_{c}(\nu)\right| \ll\left|q_{m}(\nu)\right|$ for ferrous metals and nonmagnetic coatings, the squared ratio in (19) passes from unity for $w \gg \delta_{c}$, where the coating layer dominates the reflectivity of a metal surface, to values potentially several orders of magnitude higher as $w / \delta_{c} \rightarrow 0$. The impact of the coating layer can be observed Fig. 4, where $\left|q_{a} / q_{m}(\nu)\right|$ acts as a reduction function and can be interpreted as a shielding action of the metallic coating layer that reduces interactions with the background ferrous metal. On the other hand, assuming that waves mainly interact with the coating layer, this would be interpreted as an apparent relative permeability $\mu_{a}(\nu)$, according to (20), equal to

$$
\mu_{a}(\nu)=\tilde{q}_{a}(\nu) / \sigma_{c},
$$

that would be bigger than one for $w>\delta_{c}$. This phenomenon 
is represented in Fig. 4(b), where $\mu_{a}=q_{a} / q_{c}$ represents the apparent relative permeability of the coating layer.

For the case of interest of a mild-steel surface coated with $\mathrm{Zn}$ or Fe-Zn alloys, a contrast $\left|q_{c} / q_{m}\right| \lesssim 10^{-3}$ can be expected at frequency ranges where $w \gg \delta_{c}$. Uncertainty in the actual thickness $w$, together with the variability intrinsic in the structure and composition of galvanized steel (cf. Sec. II), implies that any attempt at predicting the resulting reflectivity seems unlikely, making a compelling case for the use of the empirical procedure presented in Sec. V.

Further insight can be gained by considering some useful approximations of (19). Assuming $\left|q_{c}\right| \ll\left|q_{m}(\nu)\right|$, i.e., for a high contrast in the effective conductivities of the coating and background metals,

$$
q_{a}(\nu) \simeq q_{c} / \tanh ^{2}\left(\mathrm{j} k_{c} w\right) .
$$

This approximation is shown in Fig. 4(b), and has the advantage of not requiring prior knowledge of the magnetic permeability of the background metal used in RC walls. It is valid only as long as $\left|q_{c} / q_{m}(\nu)\right| \ll\left|\tanh \left(\mathrm{j} k_{c} w\right)\right|$, i.e., $\forall \nu \gg \nu_{1}$, with

$$
\nu_{1}=\left(2 \pi \mu_{o} \mu_{c} w^{2} \sigma_{c}^{2} q_{m}(\nu)\right)^{-1} .
$$

For the case of steel walls with $q_{m}(\nu) \simeq 10^{-3} \Omega \mathrm{m}$ and a nonmagnetic coating with $w=10 \mu \mathrm{m}, \sigma_{c}=1 \mathrm{MS} / \mathrm{m}, \nu_{1} \simeq 120$ MHz.

Over frequencies $\nu \lesssim \nu_{2}$, where $\left|k_{c} w\right| \lesssim 1$, i.e.,

$$
\nu_{2}=\left(2 \pi \mu_{o} \mu_{c} w^{2} \sigma_{c}\right)^{-1},
$$

(21) further simplifies into

$$
q_{a}(\nu) \simeq-\frac{\mathrm{j} q_{c}}{2\left(w / \delta_{c}\right)^{2}},
$$

remarkable as it is purely imaginary for the common case of non-magnetic coatings. As a consequence, (5) becomes

$$
Q_{w}(\nu) \simeq \frac{3}{2} \frac{V}{S} \pi \nu \mu_{o} w \sigma_{c},
$$

shifting the focus from steel surfaces to their coating even when $w \gg \delta_{c}$.

This last approximation predicts that there exists a frequency range where $Q_{w}(\nu)$ scales linearly with the frequency, rather than with its square root, as usually expected for homogeneous metallic surfaces. Fig. 5 shows that the transition frequency $\nu_{2}$ between these two regimes should be expected around $1 \mathrm{GHz}$ for a coating conductivity equal to $1 \mathrm{MS} / \mathrm{m}$. Clear evidence of this transition is presented in Sec. VI.

\section{EMPIRICAL CHARACTERIZATION OF RC SURFACES}

This section describes how the apparent conductivity of metallic surfaces of an RC can be estimated directly from measured data, in order to counter the difficulties summarized in Sec. II when attempting to model magnetic losses in ferrous metals.

The method calls for the average composite quality factor $Q(\nu)$ to be estimated, either with single- or double-antenna methods [37], necessarily involving a randomization by means

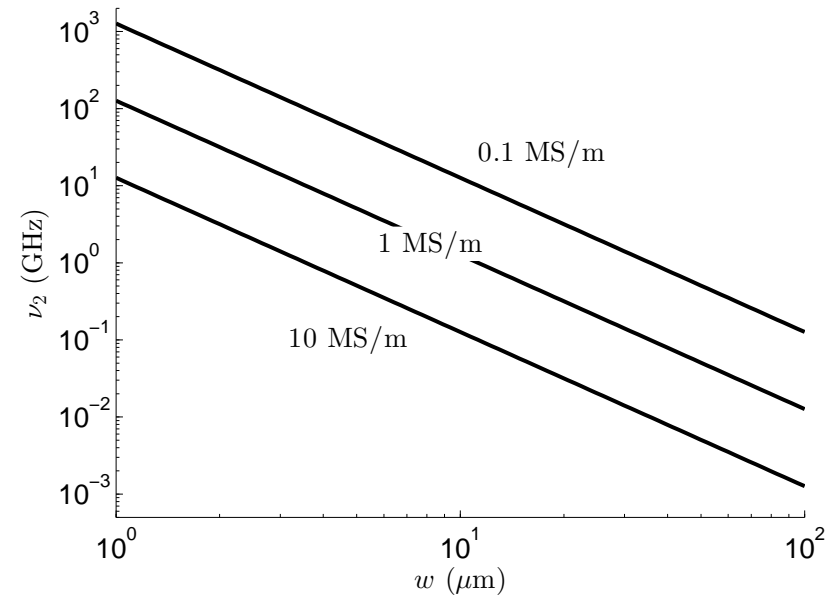

Fig. 5: Transition frequency $\nu_{2}$ around which $Q_{w}(\nu)$ is expected to pass from scaling as $\nu$ to $\nu^{1 / 2}$, as a function of the coating thickness and its conductivity.

of at least one stirring procedure [38]. The overall composite quality factor can be expressed as [1]

$$
Q^{-1}(\nu)=(N+1) Q_{a}^{-1}(\nu)+Q_{w}^{-1}(\nu),
$$

where $Q_{a}(\nu)$ and $Q_{w}(\nu)$ represent the partial contribution of antenna- and boundary-related power loss, respectively; $N$ is the number of identical antennas used during the measurement, increased by one in order to account for the fact that the excitation antenna sees twice the average received power due to coherent backscattering [39]; the $N$ antennas are assumed to be identical for the sake of simplicity.

For real antennas, with an input reflection coefficient $\Gamma_{a}(\nu)$ and radiation efficiency $\eta_{a}(\nu)$,

$$
Q_{a}=\frac{Q_{a}^{o}}{1-\eta_{a}^{2}(\nu)\left|\Gamma_{a}(\nu)\right|^{2}},
$$

as proven in [40], where

$$
Q_{a}^{o}(\nu)=16 \pi^{2} V \nu^{3} / c_{o}^{3},
$$

with $c_{o}$ the speed of light in free space. Inserting (5) and (27) into (26) yields

$$
\begin{aligned}
\frac{Q_{a}^{o}(\nu)}{Q(\nu)} & =(N+1)\left(1-\eta_{a}(\nu)^{2}\left|\Gamma_{a}(\nu)\right|^{2}\right) \\
& +\alpha S \nu^{2.5} \sqrt{\tilde{q}_{a}(\nu)},
\end{aligned}
$$

with

$$
\alpha=\frac{32 \pi^{3 / 2}}{3 c_{o}^{3} \sqrt{\mu_{o}}} .
$$

The ratio $Q(\nu) / Q_{a}^{o}(\nu)<1$ measures the average power transmission in case $N=2$ and is also known as the RC power gain. It will hereafter be referred to as $T(\nu)$.

Recalling (8), the apparent conductivity $\sigma_{a}(\nu)$ of the RC metallic surfaces can be estimated from (29) as

$$
\sigma_{a}(\nu)=\left[\frac{\alpha S \nu^{5 / 2}}{1 / T(\nu)-(N+1)\left(1-\eta_{a}^{2}(\nu)\left|\Gamma_{a}(\nu)\right|^{2}\right)}\right]^{2},
$$


which can be simplified into

$$
\sigma_{a}(\nu) \simeq \frac{\alpha^{2} S^{2} \nu^{5}}{[1 / T(\nu)-(N+1)]^{2}}
$$

as soon as the term $\eta_{a}^{2}(\nu)\left|\Gamma_{a}(\nu)\right|^{2}$ can be neglected. In order to bound the error on the estimate of the apparent conductivity $\sigma_{a}(\nu)$ to less than $10 \%,(32)$ and (31) must differ by less than $5 \%$. For well-matched antennas, with $\left|\Gamma_{a}(\nu)\right|<-10 \mathrm{~dB}$, this condition translates into

$$
T(\nu) \leq 1 / 3(N+1)
$$

which must be regarded as a worst case, since it assumes $\eta_{a}=1$.

In practice, this condition is automatically met for an overmoded RC. According to [41], if $T(\nu) \lesssim 1 / 10$, the RC can be expected to well approximate this condition with a probability of rejection $<10 \%$, if $N_{s} \geq 50$ independent random samples are available. Therefore, (32) will be employed throughout Sec. VI.

\section{EXPERIMENTAL RESULTS}

Experimental tests were carried out in CentraleSupelec, in an $\mathrm{RC}$ of dimensions $3.06 \times 1.84 \times 2.45 \mathrm{~m}^{3}$ made of steel plates. The inner volume is approximatively $13.8 \mathrm{~m}^{3}$ with a surface of $36.4 \mathrm{~m}^{2}$ covered by steel plates, including a fourplate stirrer.

The experimental setup was a standard choice for measuring the QF of an RC. It employed two identical antennas installed within the RC, one used in transmission mode and the other as a receiver. The tests covered the frequency range $0.6-16$ $\mathrm{GHz}$, using two sets of antennas: a) monocone antennas $18 \mathrm{~cm}$ wide at their top for the $0.6-6 \mathrm{GHz}$ range and b) two ridgedhorn antennas for the 6-16 GHz. The input matching was verified to be better than $-10 \mathrm{~dB}$, thus ensuring $\left|\Gamma_{a}(\nu)\right|^{2} \leq 0.1$ for all the antennas. As discussed at the end of Sec. V, knowledge of their radiation efficiency has little importance for the validation of the proposed models, thanks to their sufficient impedance matching. Previous tests on the conical antennas resulted in an efficiency higher than 0.7 over the 0.6-6 GHz range, while the ridged-horn antennas are expected to have an efficiency close to 0.9 . The two sets of antennas were placed about $2 \mathrm{~m}$ away from each other, on styrofoam supports about $1 \mathrm{~m}$ high, having taken care to have their axis orthogonal, in order to minimize any potential direct coupling. The stirrer completed a full rotation in $N_{s}=50$ steps that were checked as generating transmission samples with lag1 correlation coefficients consistent with the hypothesis of a correlation coefficient smaller than 0.1 [42], [43].

$\mathrm{S}$-matrix parameters were measured from the two antenna ports with the help of a vector network analyzer (VNA), model E8363B from Keysight, to which a full two-port correction was applied. It was operated over with a frequency resolution of $100 \mathrm{kHz}$, corresponding to roughly $1 / 4$ of the coherence bandwidth of the RC. This choice is fundamental to avoid time-aliasing when post-processing the data in time-domain. The S-matrix data were used in order to estimate the average composite QF of the RC. A time-domain approach was used

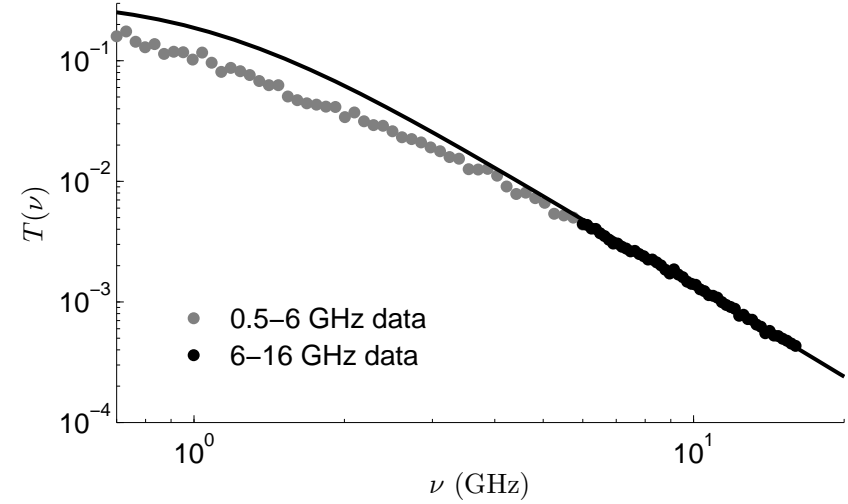

Fig. 6: Average power transmission (or gain) $T(\nu)$, defined in (29). The solid line is the theoretical prediction computed from (26) using (5) for bulk homogeneous non-magnetic walls with a conductivity of $0.95 \mathrm{MS} / \mathrm{m}$.

[44], [45], in order to avoid the uncertainties related to the correction of the residual antenna mismatch and their radiation efficiencies. This last quantity is in particular known to be affected by non-negligible uncertainties that would reduce the accuracy of the validation of the models introduced in the previous sections.

The time-domain approach estimates the $\mathrm{QF}$ from the average relaxation (or decay) time $\tau$ of the RC. This was done by first computing the power-delay profile of the RC over bandwidths of $10 \mathrm{MHz}$ and computing the best fitting regression for a decaying exponential, covering an interval of about $2 \tau$, in order to increase the accuracy of the operation; changing the bandwidth from 3 to $30 \mathrm{MHz}$ had no major impact on the results. Notice that the regression was done over the logarithm of the power-delay profile, since it is expected to present random fluctuations well-approximated by a Gaussian distribution [46].

The QF thus obtained is discussed later in this section. As suggested in Sec. V, it was transformed into the average power transmission $T(\nu)=Q(\nu) / Q_{a}^{o}(\nu)$. Fig. 6 shows the result of this operation, confirming that the RC can be considered as overmoded for $\nu>850 \mathrm{MHz}$, since $T(\nu)<1 / 10$ as discussed at the end of Sec. V. This result also implies that the radiation efficiency of the antennas has less than a $10 \%$ impact on the estimate of the apparent conductivity.

Fig. 6 also reports the theoretical prediction of $T(\nu)$ computed using (5) for bulk homogeneous non-magnetic metal walls with a conductivity of $0.95 \mathrm{MS} / \mathrm{m}$, a value chosen as it fits the high-frequency end of the experimental results. The fact that it overestimates experimental results for $T(\nu)$ in the low-frequency end suggests extra losses not explained by conductivity alone. As already discussed, a metal bulk conductivity is not expected to vary over the microwave region and surface roughness would rather present higher losses at the high-frequency end.

The apparent conductivity $\sigma_{a}(\nu)$, estimated from (32), is shown in Fig. 7. Two features are remarkable: a) $\sigma_{a}(\nu)$ presents a linear dependence to $\nu$ below $6 \mathrm{GHz}$, steeply 


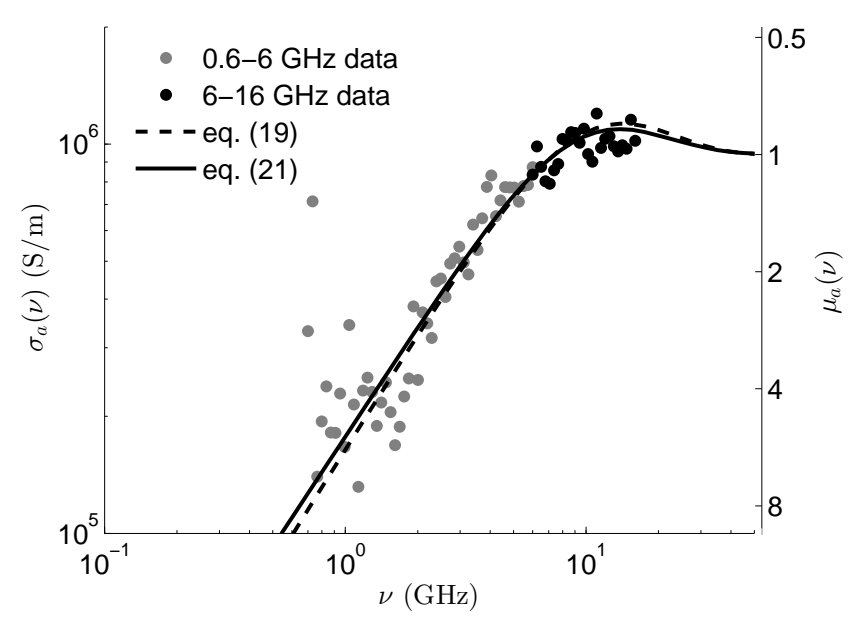

Fig. 7: Apparent conductivity $\sigma_{a}(\nu)$ obtained from the average power transmission $T(\nu)=Q(\nu) / Q_{a}^{o}(\nu)$ applying the estimator (32). A second axis on the right of the figure shows the apparent relative magnetic permeability $\mu_{a}(\nu)$ needed in order to explain the results when assuming bulk metal boundaries with $\sigma_{c}=0.95 \mathrm{MS} / \mathrm{m}$.

decreasing at lower frequency, while b) it reaches a plateau around $10 \mathrm{GHz}$. These results do not depend on the proposed layered model, since (31) and (32) are based on standard models that assume homogeneous metallic boundaries. The empirical observation of a reduction in the apparent conductivity explains the existence of extra losses at low frequencies.

The non-trivial behavior of the apparent conductivity can be explained by means of the layered model (19), which requires two parameters for the metallic coating of the plates, namely thickness $w$ and conductivity $\sigma_{c}$. These parameters were estimated by means of a least-square regression, assuming $\mu_{c} \simeq 1$ and a mild steel background (cf. Fig. 2), resulting in $w=6.8 \mu \mathrm{m}$ and $\sigma_{c}=0.95 \mathrm{MS} / \mathrm{m}$. It is important to notice that an unambiguous choice of $w$ and $\sigma_{c}$ was possible only because the results above $10 \mathrm{GHz}$ deviate from a linear slope. See Sec. VII for further discussions about this point.

The same parameters applied to the approximate model (21), for a high contrast in the effective conductivities in the wall metals, is shown as a dashed line in Fig. 7, where it is confirmed to be an effective approximation that does not rely on hard-to-get a priori information on the steel magnetic behavior.

More accurate estimates would require tests to be extended beyond $50 \mathrm{GHz}$, where $\sigma_{a}(\nu)$ is expected to converge to that of a bulk coating, according to the theoretical results shown in Fig. 7. The thickness found is consistent with typical values for electroplated steel plates for indoor applications. The coating conductivity appears to be lower than that of bulk pure zinc (about $16 \mathrm{MS} / \mathrm{m}$ ), and closer to that of bulk steel alloys (about 1.6 MS/m), in line with the arguments exposed in Sec. II-D. Similar examples of lower-than-expected conductivity can be found in [24], where a conductivity of $0.8 \mathrm{MS} / \mathrm{m}$ for surface machined cold rolled steel at $24 \mathrm{GHz}$ was reported, for which around $3 \mathrm{MS} / \mathrm{m}$ were expected at DC. An example of zinc-

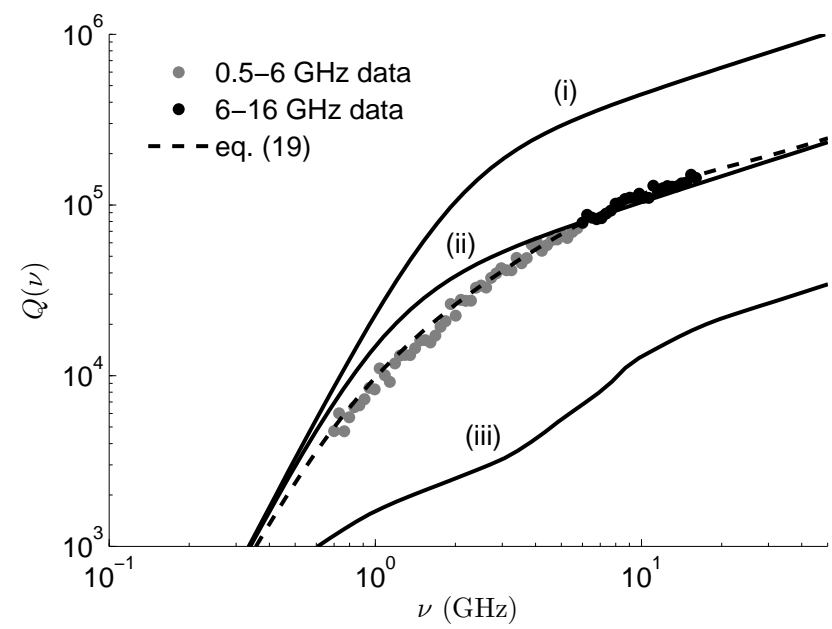

Fig. 8: Average composite QF. The three solid curves are predictions from (5) for bulk metallic boundaries, with: (i) $\sigma_{m}=16 \mathrm{MS} / \mathrm{m}, \mu_{m}=1$ (pure bulk zinc), (ii) $\sigma_{m}=0.95$ $\mathrm{MS} / \mathrm{m}, \mu_{m}=1$ and (iii) $\sigma_{m}(\nu)=1 \mathrm{MS} / \mathrm{m}, \mu_{m}$ given in Fig. 1 (bulk mild steel). The dashed curve corresponds to the layered model (19), with parameters $\sigma_{c}=0.95 \mathrm{MS} / \mathrm{m}, \mu_{c}=1$ and $w=6.8 \mu \mathrm{m}$.

plated steel with an apparent $4 \mathrm{MS} / \mathrm{m}$ conductivity measured at $8.42 \mathrm{GHz}$ was presented in [47], but no details were given about the zinc thickness and what galvanization technique was used; waves at this frequency would mostly interact with the zinc layer, with a skin depth of about $1.4 \mu \mathrm{m}$ for pure zinc.

Fig. 7 definitely rules out surface roughness as a single cause for a low apparent conductivity, since the range of values spanned by the conductivity is too wide (almost one order of magnitude) and most importantly because the apparent conductivity increases with the frequency, whereas surface roughness would have the opposite effect, as recalled in Sec. II-C. Sec. VII presents further examples taken from the literature presenting data measured in RCs that correspond to apparent conductivities even lower than $1 \mathrm{MS} / \mathrm{m}$.

The results in Fig. 7 can alternatively be interpreted by assuming bulk homogeneous materials for the metallic boundaries, with a conductivity $\sigma_{c}$. In this case, an apparent relative permeability would be attributed to the metallic surfaces, as shown on the right axis in Fig. 7. These results cannot be explained assuming that waves mainly interact with the coating layer, for which $\mu_{c}=1$ is expected. The effective permeability of mild steel could neither be used to explain this residual magnetic behavior, since it is much higher, as shown in Fig. 2. These results help to understand the fundamental role played by the metallic coating as a partial shielding of the underlying ferromagnetic material and lend direct support to the layered model introduced in Sec. IV.

The QF estimated from measured data is shown in Fig. 8 , where it is compared to theoretical predictions from (5) for different bulk metallic boundaries, with: (i) $\sigma_{m}=16$ $\mathrm{MS} / \mathrm{m}, \mu_{m}=1$ (pure bulk zinc), (ii) $\sigma_{m}=0.95 \mathrm{MS} / \mathrm{m}$, $\mu_{m}=1$ and (iii) $\sigma_{m}(\nu)=1 \mathrm{MS} / \mathrm{m}, \mu_{m}$ given in Fig. 1 (bulk mild steel). The three curves for bulk models share the 

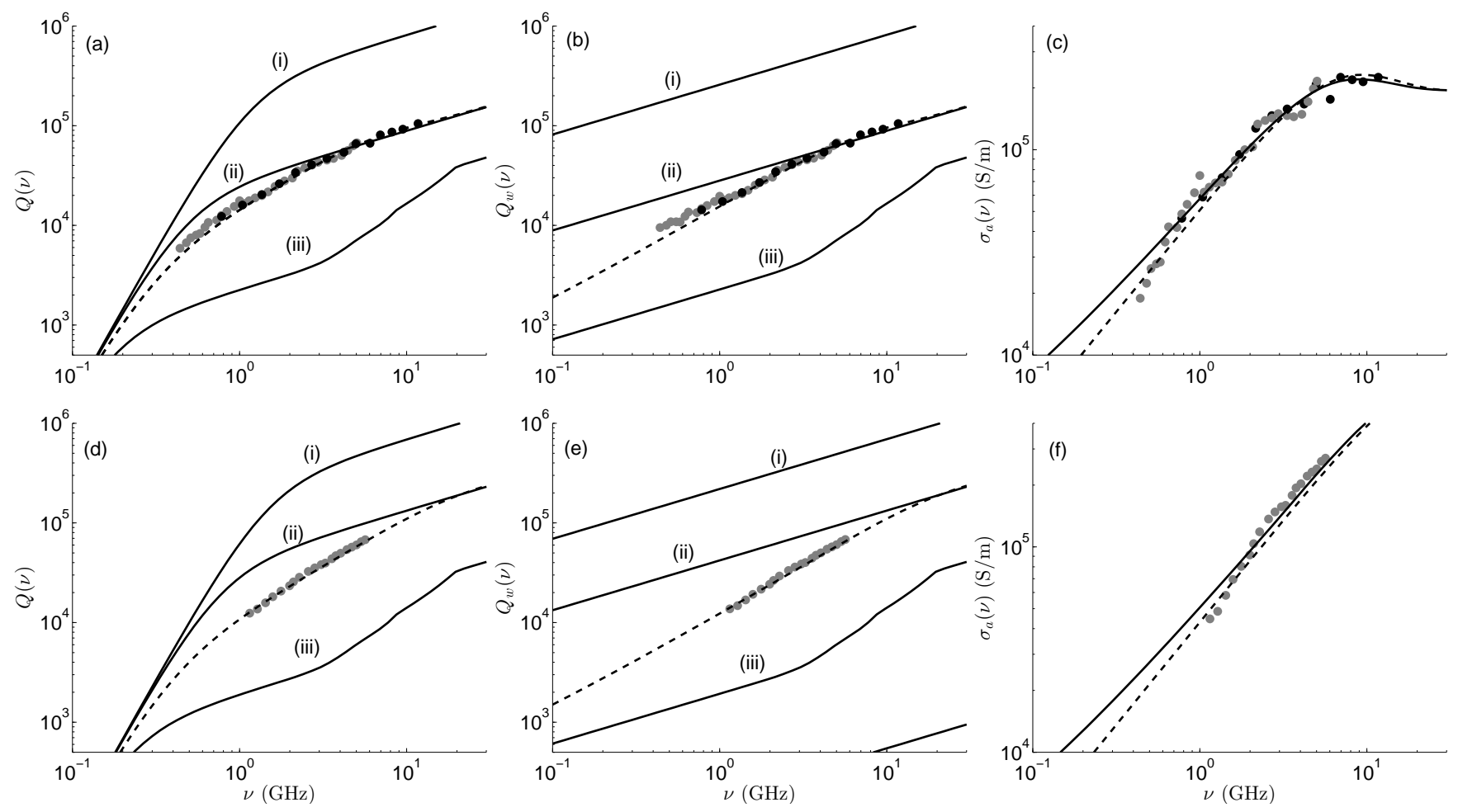

Fig. 10: Application of the proposed model to literature results from [37] (gray dots) and [48] (black dots), shown in plots (a-c) and [44] (gray dots), for plots (d-f). The original results were averaged over bandwidths about $200 \mathrm{MHz}$ wide in order to ease comparisons with theory. Refer to Figs. 7 and 8 for the meaning of solid and dashed lines and Sec. VII for details about model parameters.

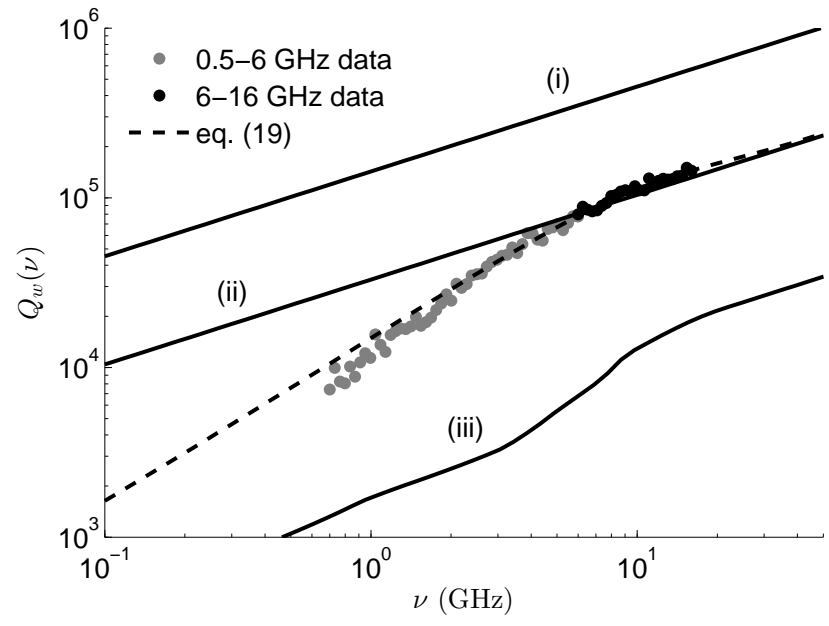

Fig. 9: Wall-dissipation contribution $Q_{w}(\nu)$ to the QF, estimated from measured data by means of (34). Refer to Fig. 8 for the meaning of curves (i)-(iii).

$\nu^{1 / 2}$ dependence typically expected, which is supported by the experimental results only above $10 \mathrm{GHz}$ for $\sigma_{m}=0.95$ $\mathrm{MS} / \mathrm{m}$; the results for configurations (i) and (iii) are almost one order of magnitude off the experimental results. At lower frequencies $Q(\nu)$ moves to the $\nu^{3}$ behavior expected for antenna-dominated losses. Discrepancies are visible below 6
$\mathrm{GHz}$ between curve (ii) and experimental results; curves (i) and (iii) are clearly unable to explain the results, confirming the inadequacy of bulk metal models. In particular, interactions limited to the coating layer, expected above $10 \mathrm{GHz}$, would be systematically overestimated assuming a pure zinc layer (cf. Sec. II-D). On the contrary, predictions from the proposed layered model closely agree over the entire frequency range.

The accuracy of the proposed model can be better appreciated in Fig. 9, where the wall-dissipation contribution $Q_{w}(\nu)$ to the composite QF is shown, estimated from (26) as

$$
Q_{w}(\nu)=\left[Q^{-1}(\nu)-3 Q_{a}^{-1}(\nu)\right]^{-1} .
$$

Fig. 9 clearly proves the existence of two regimes for $Q_{w}(\nu)$ : below $6 \mathrm{GHz} Q_{w}(\nu) \sim \nu$, as predicted by (25), while $Q_{w}(\nu) \sim \nu^{1 / 2}$ above $6 \mathrm{GHz}$, as expected for bulk non-magnetic metals. The proposed layered model accurately reproduces the transition between these two responses observed in the experimental results, based on the two effective parameters $w$ and $\sigma_{c}$.

\section{FURTHER RESULTS FROM THE LITERATURE}

The accuracy of the proposed model was further tested by applying it to results available in RC literature. Data measured at IETR, France, have been presented in several papers, but those in [37] were chosen as they were cross-checked by using a number of estimators, thus more reliable than singleconfiguration test campaigns. Even though they are limited to 
about $5 \mathrm{GHz}$, similar measurements were carried out anew up to $12 \mathrm{GHz}$ in [48], for a similar configuration. The two data sets are shown in Figs. 10(a)-(c), and closely overlap over a large frequency range. Data below $450 \mathrm{MHz}$ were excluded as they did not fulfill condition (33), for which antenna radiation efficiencies are not needed when estimating the apparent conductivity $\sigma_{a}(\nu)$.

As in the previous section, the proposed layered model was fitted to the data, resulting in $w=18.5 \mu \mathrm{m}$ and $\sigma_{c}=0.195$ $\mathrm{MS} / \mathrm{m}$. The thickness is compatible with both electroplated or hot-dip galvanized steel plates (cf. Sec. II-D). Similar low values of conductivity were already tentatively used in [48], using a bulk metal model; similar results of apparent conductivity were reported in [47]. Furthermore, it is noteworthy to recall how the Authors of [48] expressed what appears to be surprise at the very slow convergence of $Q(\nu)$ to the $\nu^{1 / 2}$ dependence expected from bulk models, since a linear frequency dependence fitted more accurately to describe their results. Also these results feature a transition between two regimes in $Q_{w}(\nu)$, with the transition occurring around $5 \mathrm{GHz}$ in Fig. 10(b). The apparent conductivity decreases at lower frequencies to values as low as $0.02 \mathrm{MS} / \mathrm{m}$, explaining the extra losses observed in Fig. 10(a) below $3 \mathrm{GHz}$.

A second case from the literature is taken from [44], with data measured in NIST's RC. A number of papers report data measured in this historical chamber, but those in [44] stand out as they where obtained using a time-domain approach for the estimate of $Q(\nu)$, thus avoiding the issue of correcting for non-ideal antennas [44], [45]. This data set spans a limited frequency range, approximatively going from 1 to $7 \mathrm{GHz}$.

The results obtained with this data set are shown in Figs. 10(d)-(f). In this case the apparent conductivity does not converge to the fixed value representing the effective coating conductivity. It is therefore not possible to find a unique set of optimal $w$ and $\sigma_{c}$ fitting the data. Instead, an optimal product $w \sigma_{c}=3.3 \mathrm{~S}$ was found. The theoretical results in Figs. 10(d)(f) were obtained choosing $w=6 \mu \mathrm{m}$ and $\sigma_{c}=0.55 \mathrm{MS} / \mathrm{m}$. Independently from this choice, the linear regime for $Q_{w}(\nu)$ is experimentally confirmed also in this case.

\section{DISCUSSIONS AND CONCLUSIONS}

The introduction of an estimator of the apparent conductivity of an RC metallic boundaries has provided unambiguous evidence that bulk descriptions are inaccurate for the case of steel plates. The inherent dispersion of the apparent conductivity explains why the $\nu^{1 / 2}$ dependency of the composite QF may not appear as accurate below about $10 \mathrm{GHz}$.

The brief review of steel-plate properties in Sec. II was intended to help understand the high variability of parameters related to ferrous materials. Arguably, the acknowledgement of the complex nature of their magnetic permeability, jointly with the introduction of the coating-layer model, is the most important result in this paper, since it provides a reasonable explanation for the extra losses observed below a few $\mathrm{GHz}$ and the slow convergence of the composite QF to a $\nu^{1 / 2}$ dependence.

A question left open by this paper is the surprisingly low values found for the coating conductivity, well below that of steel. It seems appropriate to recall that this is in fact an apparent conductivity. The assumption of a non-magnetic coating is not necessarily accurate, e.g., in case of inter-layer diffusion of iron, as discussed in Sec. II-D. As such, even weak magnetic responses from the coating would result in a lower apparent conductivity. This conjecture would need further tests in order to confirm its accuracy and is left for future work.

The coating-layer model has direct application in RC designs, e.g., in order to estimate the minimum coating thickness $w_{\text {min }}$ needed in order to avoid extra losses from ferrous materials. An example is presented in Fig. 11, where results were computed by forcing a loss of 1 or $3 \mathrm{~dB}$ in the power gain $T(\nu)$ in (29) between the case of a bulk metal with conductivity $\sigma_{c}$ and a steel plate with a coating of the same material, but of finite thickness $w_{\min }$; a total metallic surface $S_{o}=100 \mathrm{~m}^{2}$ was considered, together with the presence of two well-matched antennas. The minimum thickness peaks about $1 \mathrm{GHz}$, and rapidly decreases at lower frequencies where antenna-related losses take effect, as well at higher frequencies where dissipation within the coating layer reduces interactions with the background steel. The results in Fig. 11 are independent of the RC volume and can be extended to other configurations for a surface $S$ and frequency $\nu$ by looking up for

$$
\nu_{o}=\nu\left(S / S_{o}\right)^{2 / 5} .
$$

These results show that there is not need to use expensive solutions based on non-ferromagnetic bulk metal plates made of, e.g., aluminum, brass or copper. Instead, these metals could be used as thin coatings that would be effective in avoiding extra losses with just a thickness of a few microns. Steel plates would then only contribute to mechanical stiffness, while waves would mostly interact with thin non-ferromagnetic coatings.

Notice that the minimum thickness estimated in Fig. 11 is not overly conservative, since it is not necessarily larger than the penetration depth. In our investigations zinc coatings, potentially subject to iron diffusion, appear to lead to apparent conductivities below $1 \mathrm{MS} / \mathrm{m}$, even above $10 \mathrm{GHz}$, where interactions with background steel can be expected to be negligible, thus leaving room for improvement by using better conducting metals less prone to iron diffusion.

\section{REFERENCES}

[1] D. Hill, "Electromagnetic Theory of Reverberation Chambers," National Institute of Standards and Technology, Tech. Rep. 1506, 1998.

[2] M. Crawfordl and G. Koepke, "Design, Evaluation and Use of a Reverberation Chamber for Performing Electromagnetic Susceptibility/Vulnerability Measurements,' National Bureau of Standards, Tech. Rep. 1092, 1986.

[3] P. Besnier, J. Sol, A. Presse, C. Lemoine, and A. C. Tarot, "Antenna efficiency measurement from quality factor estimation in reverberation chamber," in 46th European Microwave Conference (EuMC), Oct 2016, pp. 715-718.

[4] "Infonotes of the American Galvanizers Association," http://www.galvinfo.com/galvinfonotes/.

[5] C. W. Burrows, "Correlation of the magnetic and mechanical properties of steel," Bulletin of the Bureau of Standards, vol. 13, no. 2, pp. 176210, 1916.

[6] J. Weibler, "Properties of metals used for RF shielding," EMC Test and Design, p. 100, 1993.

[7] H. W. Ott, Electromagnetic compatibility engineering. John Wiley \& Sons, 2011. 


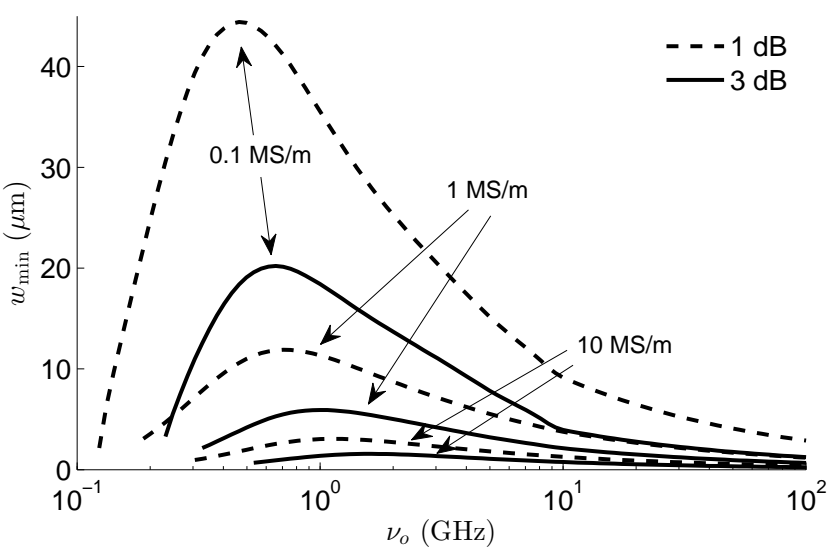

Fig. 11: Minimum coating thickness $w_{\min }$ required such that extra losses for steel plates be limited to 1 or $3 \mathrm{~dB}$, with respect to bulk metallic surfaces of conductivity $\sigma_{c}$ (for the metallic coating). Results for three values of $\sigma_{c}$ are shown in the graph, computed for an RC of internal surface $S_{o}=100 \mathrm{~m}^{2}$; cf. (35) to apply these results to other configurations.

[8] D. Morgan, A Handbook for EMC Testing and Measurement. IET, 1994, vol. 8.

[9] B. E. Keiser, Principles of electromagnetic compatibility. Artech House, 1979.

[10] D. Weston, Electromagnetic Compatibility: Principles and Applications, Revised and Expanded. CRC Press, 2001.

[11] C. R. Paul, Introduction to electromagnetic compatibility. John Wiley \& Sons, 2006, vol. 184.

[12] V. P. Kodali, Engineering electromagnetic compatibility: principles, measurements, technologies, and computer models. IEEE, 2001.

[13] M. Houlden and P. Chatteron, EMC Electromagnetic Theory to practical design. John Wiley and Sons, New York, 1992.

[14] F. M. Tesche, M. Ianoz, and T. Karlsson, EMC analysis methods and computational models. John Wiley \& Sons, 1997.

[15] J. M. Ladbury, G. H. Koepke, and D. G. Camell, "Evaluation of the NASA langley research center mode-stirred chamber facility, Tech. Rep. Technical Note (NIST TN)-1508, 1999.

[16] F. Benson, "Attenuation in nickel and mild-steel waveguides at 9375 $\mathrm{mc} / \mathrm{s}$," Proceedings of the IEE-Part III: Radio and Communication Engineering, vol. 101, no. 69, pp. 38-41, 1954.

[17] J. Allison, F. Benson, and M. Seaman, "Characteristics of some ferrous and non-ferrous waveguides at $27 \mathrm{gc} / \mathrm{s}$," Proceedings of the IEE-Part B: Radio and Electronic Engineering, vol. 104, no. 18, pp. 599-602, 1957.

[18] R. Millership and F. V. Webster, "High frequency permeability of ferromagnetic materials," Proceedings of the Physical Society. Section $B$, vol. 63, no. 10, p. 783, 1950.

[19] C. Kittel, "Theory of the dispersion of magnetic permeability in ferromagnetic materials at microwave frequencies," Physical Review, vol. 70, no. 5-6, p. 281, 1946.

[20] J. Allanson, "The permeability of ferromagnetic materials at frequencies between $10^{5}$ and $10^{10} \mathrm{c} / \mathrm{s}, "$ Journal of the Institution of Electrical Engineers-Part III: Radio and Communication Engineering, vol. 92, no. 20, pp. 247-255, 1945.

[21] K. N. Rozanov and M. Y. Koledintseva, "Limitations on high-frequency permeability of magnetic materials," in Electromagnetic Compatibility (EMC), 2013 IEEE International Symposium on. IEEE, 2013, pp. 551556.

[22] G. F. Hodsman, G. Eichholz, and R. Millership, "Magnetic dispersion at microwave frequencies," Proceedings of the Physical Society. Section B, vol. 62, no. 6, p. 377, 1949. [Online]. Available: http://stacks.iop.org/0370-1301/62/i=6/a=305

[23] C. Graham Jr, "Physical origin of losses in conducting ferromagnetic materials," Journal of Applied Physics, vol. 53, no. 11, pp. 8276-8280, 1982.

[24] E. Maxwell, "Conductivity of metallic surfaces at microwave frequencies," Journal of Applied Physics, vol. 18, no. 7, pp. 629-638, 1947.
[25] S. P. Morgan, "Effect of surface roughness on eddy current losses at microwave frequencies," Journal of Applied Physics, vol. 20, no. 4, pp. 352-362, 1949.

[26] "Zinc coatings. Guidelines and recommendations for the protection against corrosion of iron and steel in structures. General principles of design and corrosion resistance," International Organization for Standardization," Standard, 2017.

[27] "Zinc coatings," https://www.galvanizeit.org/corrosion/corrosionprotection/zinc-coatings.

[28] "Zinc coatings and applications chart," https://www.galvanizeit.org/knowledgebase/article/zinc-coatings-andapplications-chart.

[29] Americal Welding Society, "Inspecting welds in galvanized steel," https://app.aws.org/itrends/2005/10/023/.

[30] G. Oluwadare, "Evaluation of influence of process variables on the corrosion performance of hot-dip-galvanized steel sheets," Academic Journals Trends in Applied Sciences Research, vol. 2, no. 4, pp. 320326, 2007.

[31] G. Boato, M. Bugo, and C. Rizzuto, "The effect of transition-metal impurities on the residual resistivity of $\mathrm{Al}, \mathrm{Zn}, \mathrm{In}$ and $\mathrm{Sn}$," Il Nuovo Cimento B (1965-1970), vol. 45, no. 2, pp. 226-240, Oct 1966.

[32] I. H. Karahan, "A study on electrodeposited $\mathrm{Zn}_{1-x} \mathrm{Fe}_{x}$ alloys," Journal of Materials Science, vol. 42, no. 24, pp. 10 160-10 163, 2007.

[33] K. Sumiyama and Y. Nakamura, "Magnetic properties of Fe-Zn alloys produced by vapor quenching," IEEE Translation Journal on Magnetics in Japan, vol. 1, no. 9, pp. 1099-1100, Dec 1985.

[34] J. Van Bladel, Electromagnetic fields. IEEE, 2007.

[35] E. P. Wohlfarth, Ferromagnetic materials: a handbook on the properties of magnetically ordered substances. Elsevier, 1980, vol. 2.

[36] J. Dobrowolski, Microwave network design using the scattering matrix. Artech House, 2010.

[37] P. Besnier, C. Lemoine, and J. Sol, "Various estimations of composite q-factor with antennas in a reverberation chamber," in 2015 IEEE International Symposium on Electromagnetic Compatibility (EMC), Aug 2015, pp. 1223-1227.

[38] R. Serra, A. C. Marvin, F. Moglie, V. M. Primiani, A. Cozza, L. R. Arnaut, Y. Huang, M. O. Hatfield, M. Klingler, and F. Leferink, "Reverberation chambers a la carte: An overview of the different modestirring techniques," IEEE Electromagnetic Compatibility Magazine, vol. 6, no. 1, pp. 63-78, First 2017.

[39] I. Junqua, P. Degauque, M. LiẪl'nard, and F. Issac, "On the power dissipated by an antenna in transmit mode or in receive mode in a reverberation chamber," IEEE Trans. Electromagn. Compat., vol. 54, no. 1, pp. 174-180, Feb 2012.

[40] A. Cozza, "Power loss in reverberation chambers by antennas and receivers," IEEE Transactions on Electromagnetic Compatibility, pp. 14, 2017

[41] _ - "Likelihood of meeting isotropic conditions in reverberation chambers from power transmission," IEEE Antennas Wireless Propag. Lett., vol. 16, pp. 2881-2884, 2017.

[42] R. A. Fisher, "Frequency distribution of the values of the correlation coefficient in samples from an indefinitely large population," Biometrika, vol. 10 , no. 4, pp. 507-521, 1915.

[43] J. S. White, "Asymptotic expansions for the mean and variance of the serial correlation coefficient," Biometrika, vol. 48, no. 1-2, pp. 85-94, 1961. [Online]. Available: http://dx.doi.org/10.1093/biomet/48.1-2.85

[44] C. L. Holloway, H. A. Shah, R. J. Pirkl, W. F. Young, D. A. Hill, and J. Ladbury, "Reverberation chamber techniques for determining the radiation and total efficiency of antennas," IEEE Transactions on Antennas and Propagation, vol. 60, no. 4, pp. 1758-1770, April 2012.

[45] J. C. West, J. N. Dixon, N. Nourshamsi, D. K. Das, and C. F. Bunting, "Best practices in measuring the quality factor of a reverberation chamber," IEEE Transactions on Electromagnetic Compatibility, vol. 60, no. 3, pp. 564-571, 2018.

[46] M. S. Bartlett and D. Kendall, "The statistical analysis of varianceheterogeneity and the logarithmic transformation," Supplement to the Journal of the Royal Statistical Society, vol. 8, no. 1, pp. 128-138, 1946.

[47] T. Y. Otoshi and M. M. Franco, "The electrical conductivities of steel and other candidate materials for shrouds in a beam-waveguide antenna system," IEEE Transactions on Instrumentation and Measurement, vol. 45, no. 1, pp. 77-83, Feb 1996.

[48] L. Arnaut, P. Besnier, J. Sol, and M. Andries, "On the uncertainty quantification of the quality factor of reverberation chambers," IEEE Trans. Electromagn. Compat., vol. 99, 2018. 